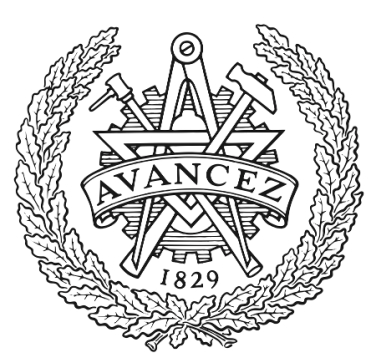

\title{
CHALMERS
}

UNIVERSITY OF TECHNOLOGY

\section{Integrated W-band Photoconductive Switches in SIW Technology}

Downloaded from: https://research.chalmers.se, 2023-04-26 09:48 UTC

Citation for the original published paper (version of record):

Shepeleva, E., Makurin, M., Evtyushkin, G. et al (2021). Integrated W-band Photoconductive Switches in SIW Technology. IEEE Microwave and Wireless Components Letters, 31(7): 865-868. http://dx.doi.org/10.1109/LMWC.2021.3075347

N.B. When citing this work, cite the original published paper.

C2021 IEEE. Personal use of this material is permitted.

However, permission to reprint/republish this material for advertising or promotional purposes 


\title{
Integrated W-band Photoconductive Switches in SIW Technology
}

\author{
Elena Shepeleva, Mikhail Makurin, Gennady Evtyushkin, Anton Lukyanov, Artem Vilenskiy, \\ Member, IEEE, Sergey Chernyshev, and Marianna Ivashina, Senior Member, IEEE
}

\begin{abstract}
A novel approach for the design of mmWave substrate integrated waveguide (SIW) switches is presented. The switch is based on controllable photoconductive elements (PEs), whose conductivity is modulated by a light source. The PE is integrated on the top SIW metal plate and provides either a shortcircuit or transmission operation regime, depending on the actuation conditions. Owing to the light actuation, the mmWave and DC control circuitry are naturally decoupled, which, in turn, makes the design low-loss and compact as compared to PIN diode and integrated circuit-based SIW switches implementations. We demonstrate two examples of SIW photoconductive switches (PSs) at W-band: single-pole single-throw (SPST) and single-pole double-throw (SPDT) PSs. Their operation principle, actuation, and parasitic radiation suppression are discussed. Measurements show the peak insertion loss of 0.9 and $2.2 \mathrm{~dB}$, isolation better than 25 and $30 \mathrm{~dB}$, and -10-dB impedance bandwidth of 21 and $15 \%$ for the SPST and SPDT PSs, respectively.
\end{abstract}

Index Terms - substrate integrated waveguide, photoconductive switch, single-pole double-throw, W-band.

\section{INTRODUCTION}

$\mathrm{R}$ esently, new commercial applications of millimeter-wave (mmWave) frequency wireless technologies have emerged [1] - [3]. Among them are automotive radars [1], [2], telecommunications [3], and imaging systems operating from $\mathrm{K}$ - to W-bands. These applications typically use multiple-input multiple-output (MIMO) active antenna solutions to meet the demanding requirements on high spatial resolution and wide beamsteering range [4]. Since the number of active front-end channels is usually limited, as e.g. in the case of automotive radar monolithic microwave integrated circuits (MMIC), the high resolution can be achieved through time-domain multiplexing by switching between multiple radiating elements [5]. In such systems, mmWave switches play a key role in realizing the desired beamsteering/beamforming flexibilities. Today, W-band switches are usually implemented in unified and affordable MMIC technologies [6], [7]. However, their application is limited to microstrip/strip lines and grounded coplanar waveguides (GCWG) due to the packaging and

Manuscript received 16.12.20; Revised 04.04.21; accepted 17.04.21. Date of publication XX.XX.XX. This research was supported by Samsung Research Institute Russia with an in-kind contribution (A. Vilenskiy and M. Ivashina) to the ChaseOn Centre in Integrated Antenna Array project financed by Vinnova, Chalmers University of Technology, KTH, Ericsson, Saab, Ruag Space, Keysight, and Gapwaves.

E. Shepeleva is with Samsung Research Institute Russia, Moscow, 127018, Russia, and with the Department of Radio-Electronic Systems and Devices, required DC lines tracing. On the other hand, the substrate integrated waveguide (SIW) technology has recently demonstrated the capability of providing high-efficient wideband solutions for W-band array antennas [8], [9]. Since an MMIC can be integrated with the SIW only through additional transitions [10], such implementations usually result in bulky and lossy designs. SIW switches based on PIN diodes have been reported recently at X-and K-bands [11], [12]. As the PIN diodes employ the DC control, such SIW switches require DC biasing circuitry with decoupling filters. This results in complicated assemblies with large footprints, increased insertion loss, and low isolation. An X-band SIW ferrite-loaded switch was recently presented in [13], demonstrating relatively low insertion loss, however, at a cost of large longitudinal size.

The idea of using a photoconductive element (PE) semiconductor structure, whose conductivity is modulated by a light source - has been employed recently for the development of photoconductive switches (PSs) in microstrip [14], [15] and GCWG topologies [16], as well as for reconfigurable antennas [17], [18]. More recently, we reported the first SIW PSs at Kband [19]. The seamless integration, low losses, and essential decoupling of control and mmWave circuitry are the main advantages of such SIW PS implementations. The novel contribution of this work is the scalability of the SIW PS technology to higher frequencies, in particular at W-band, where the insertion losses, parasitic radiation, and the printed circuit board (PCB) manufacturing limitations become critical. These extra complexities of the higher frequencies call for innovative solutions with external PE shielding that have been herein demonstrated through single-pole single-throw (SPST) and single-pole double-throw (SPDT) SIW PS design examples.

\section{SWITCH OPERATION PRINCIPLE}

\section{A. Photoconductive Element}

The photoconductive effect [20] is exploited to actuate the PE: the increase in electrical conductivity $(\Delta \sigma)$ of the semiconductor material occurs upon absorption of the optical

Bauman Moscow State Technical University, Moscow, 105005, Russia (e-mail: e.shepeleva@samsung.com).

M. Makurin, G. Evtyushkin, and A. Lukyanov are with Samsung Research Institute Russia.

A. Vilenskiy and M. Ivashina are with the Electrical Engineering Department, Chalmers University of Technology, Gothenburg 41258, Sweden.

S. Chernyshev is with the Department of Radio-Electronic Systems and Devices, Bauman Moscow State Technical University. 
source radiation (with power $P_{\text {opt }}$ ). There is a simplified relationship between the $P_{\text {opt }}$ and $\Delta \sigma$ (see (1) in [19]). In this work, the intrinsic silicon wafer was used to provide high PE resistivity $(\sim 7 \mathrm{k} \Omega \cdot \mathrm{cm})$ in the non-actuated state. The top and bottom wafer surfaces were passivated by a $20 \mathrm{~nm} \mathrm{Al}_{2} \mathrm{O}_{3}$ film [21]. This deposited film increases the effective minority carrier lifetime $\tau_{\text {eff. }}$ As a result, $P_{\text {opt }}$ can be significantly reduced to reach the required $\Delta \sigma$. The effective actuation of the intrinsic silicon can be realized in the near infra-red spectrum [19] when providing the full optical power absorption by the PE volume. In the present work, the wafer thickness is $160 \mu \mathrm{m}$. It was found that a light-emitting diode (LED) with $940 \mathrm{~nm}$ wavelength can effectively actuate the PE volume: light penetration depth is $\sim 100 \mu \mathrm{m}$, and the $160-\mu \mathrm{m}$ thick PE absorbs more than $85 \%$ of the power accepted by its surface, while the surface reflection coefficient is 0.3 [19]. The measured $\tau_{\text {eff }}$ of the passivated PE is $15 \mu \mathrm{s}$, which defines the OFF-ON and ON-OFF PS switching times of 20 and $40 \mu \mathrm{s}$, respectively. Thus, according to [19], $100 \mathrm{~mW}$ of DC power is required to get $\Delta \sigma=150 \mathrm{~S} / \mathrm{m}$ for a $1 \times 1 \mathrm{~mm}^{2} \mathrm{PE}$ and $40 \%$ LED conversion efficiency.

\section{B. An SIW Switching Building Block}

Figure 1 presents the 3D geometry of an SIW switching building block. The PCB design is similar to the one in [19], where the shunt VIA with radius $R_{c}$ connects the bottom PCB ground and the pad with radius $R_{P A D}$ on the top layer. The pad is separated from the top SIW metal plate by the annular slot with width $W_{s}$. The PE is placed on the top PCB layer and fully covers the slot. An equivalent circuit of the device is given in Fig. 1(a). When actuated (ON-state), the PE shunts the slot $\left(R_{P E}\right.$ becomes low), and thus a small VIA inductance $L_{V I A}$ connects the top and bottom SIW metal plates leading to the reflection of the incident SIW wave. The value of $R_{P E}$ largely determines the dissipative loss of the reflected wave. In the non-actuated (OFFstate), the impedance matching with the input SIW line is realized by adjusting the annular slot length to make it equal to the wavelength of the inhomogeneous slot line at the central design frequency $(79 \mathrm{GHz})$. In this case, as shown in Fig. 2(a), $L_{P E}$ and $C_{P E}$ of the PE-covered slot structure resonate, realizing the zero-current condition on the central VIA that effectively mitigates its shunting effect. As a result, no additional matching VIAs are required ( $c f$. [19]). In the OFF-state, a high value of the intrinsic silicon $R_{P E}$ facilitates low insertion loss. However, since the slot has relatively large electrical dimensions, a significant radiation loss typically occurs (high radiation conductance $\left.1 / R_{R A D}\right)$. To mitigate this unwanted radiation effect, we have covered the PE by the square waveguide (WG) section with the height $H_{W G}$ [Fig. 1(b)]. The WG transverse size $W_{W G}=1.3 \mathrm{~mm}$ is chosen to conveniently accommodate the $1 \times 1 \mathrm{~mm}^{2} \mathrm{PE}$ and to provide the below-cut-off WG operation regime (thus, effectively increasing $R_{R A D}$ ). Based on the fullwave simulations in Ansys HFSS [Fig. 2(b)], the WG with $H_{W G}=1 \mathrm{~mm}$ reduces the relative radiation level from $-6 \mathrm{~dB}$ (no box) to $-18 \mathrm{~dB}$ at $79 \mathrm{GHz}$, thus minimizing the insertion loss. These WG parameters are used in the following sections. The WG is formed inside a metal box, which has an additional top cavity segment for the LED. In this study, we used the Lumileds L1IZ-0940 LED that was mounted on a supporting PCB (providing DC control) and inserted into the box.

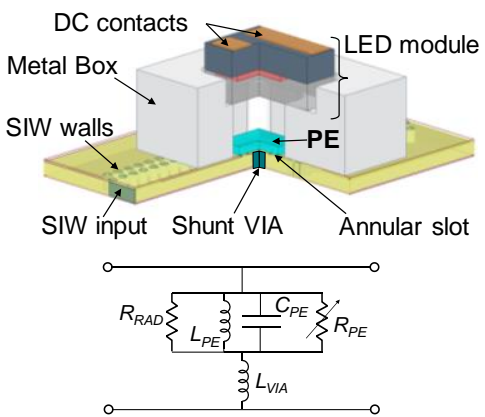

(a)

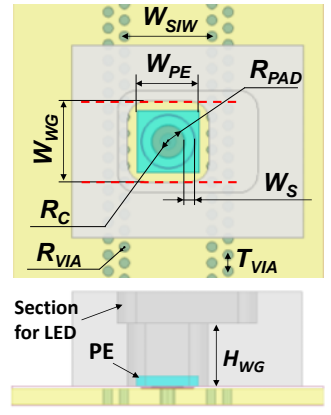

(b)
Fig. 1. Basic SIW switching block (SPST): (a) cross-section view (the equivalent circuit is given in the bottom); (b) detailed views with design parameters.

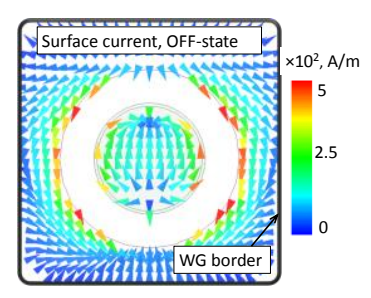

(a)



(b)
Fig. 2. (a) Surface current distribution below the PE (OFF-state) at $79 \mathrm{GHz}$. (b) Relative radiated power for different waveguide heights $\left(H_{W G}\right)$.

\section{DESIGN OF SIW PhOtOCONDUCTIVE SWITCHES}

\section{A. SPST Photoconductive Switch}

The above-described building block is used for the W-band SPST PS design with $79 \mathrm{GHz}$ as the central frequency. The SPST PS, as presented in Fig. 1, has been optimized through a full-wave electromagnetic model in Ansys HFSS. In this model, the PE element was considered as a silicon brick with $W_{P E}=$ $1 \mathrm{~mm}$. In the actuated state, the silicon conductivity was set as $100 \mathrm{~S} / \mathrm{m}$, which was found to be a sufficient value to realize a high SPST isolation [19]. For the PCB, we used a 0.127-mm thick Rogers RO3003 substrate $\left(\varepsilon_{r}=3, \tan \left(\delta_{e}\right)=0.0013\right)$. The SIW line has the following dimensions: $W_{S I W}=1.4 \mathrm{~mm}, T_{V I A}=$ $0.26 \mathrm{~mm}$, sidewall VIA radius $R_{V I A}=0.08 \mathrm{~mm}$. Other parameters were optimized for the minimum insertion loss and the return loss of less than $10 \mathrm{~dB}$ in the $15 \%$ relative bandwidth. The final design parameters are: $R_{P A D}=0.27 \mathrm{~mm}, W_{s}=$ $0.15 \mathrm{~mm}, R_{c}=0.125 \mathrm{~mm}$.

During the fabrication of the experimental prototype, the metal box was milled from aluminum and mounted onto the SIW PCB along with the PE using silver-filled epoxy adhesive. The acceptable PE misalignment is $80 \mu \mathrm{m}$, which provides that the slot is fully covered by the PE. The assembled prototype is presented in Fig. 3(a). During measurements, the LED DC power was adjusted to reach the expected insertion loss. Eventually, the DC power was set to $150 \mathrm{~mW}$ (60 mA), which is higher as compared to the value predicted by the model in [19]. This can be attributed to (i) an optical power loss due to PE and LED apertures mismatch; (ii) WG walls surface roughness; (iii) a nonuniform PE conductivity distribution [21]. Figs. 3(c) and 3(d) compare the simulated and measured $S$ parameters. Note that we used a TRL calibration setup to shift 




(a)

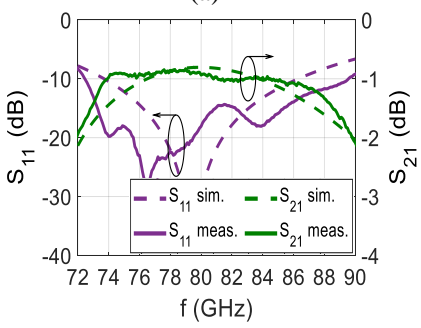

(c)

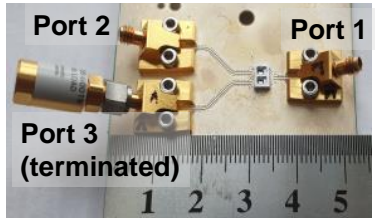

(b)



(d)
Fig. 3. Fabricated prototypes of (a) the SPST and (b) SPDT PSs (the LEDs and supporting PCBs were removed). Comparison of SPST PS measured and simulated $S$-parameters in the (c) OFF- and (d) ON-state.

the measurement reference planes to the edges of the PE [red lines in Fig. 1(a)]. The measurement results demonstrate a $72.5-89.5 \mathrm{GHz}-10-\mathrm{dB}$ impedance bandwidth, with the peak insertion loss of $0.9 \mathrm{~dB}$ (OFF-state) and isolation better than $25 \mathrm{~dB}$ (ON-state).

\section{B. SPDT Photoconductive Switch}

The SPDT PS design is demonstrated in Fig. 4(a). As seen, the SPDT PS is based on the folded H-plane SIW T-junction and two SPST blocks connected to the input port [port 1, Fig. 4(a)] at a distance L. Ideally, the SPST blocks can be integrated directly into the T-junction area. However, this significantly complicates the manufacturing of the shielding box due to a small separation between PEs. Three matching VIAs are used to compensate for the T-junction discontinuity.

The SPDT experimental prototype was developed using $0.203-\mathrm{mm}$ thick Rogers RO4003C substrate $\left(\varepsilon_{r}=3.55\right.$, $\left.\tan \left(\delta_{e}\right)=0.0027\right)$; SIW line geometry: $W_{S I W}=1.45 \mathrm{~mm}, T_{V I A}=$ $0.45 \mathrm{~mm}, R_{V I A}=0.1 \mathrm{~mm}$. The following design parameters were found during the full-wave optimization: $R_{P A D}=0.25 \mathrm{~mm}$, $W_{s}=0.15 \mathrm{~mm}, R_{c}=0.15 \mathrm{~mm}, D=2.24 \mathrm{~mm}, L=2.89 \mathrm{~mm}$. The simulated SPDT E-field distribution is demonstrated in Fig. 4(b) ( $\mathrm{PE}_{1}$ is in the OFF-state, $\mathrm{PE}_{2}$ is in the $\mathrm{ON}$-state).

The fabricated prototype is shown in Fig. 3(b). Measurements were realized in the 2-port regime: ports 1 and 2 were connected to a vector network analyzer, while port 3 was terminated with a matched load. Reference planes are shown in Fig. 4(a). The simulated and measured $S$-parameters are in good agreement, as seen in Fig. 5. The SPDT PS demonstrates $74-$

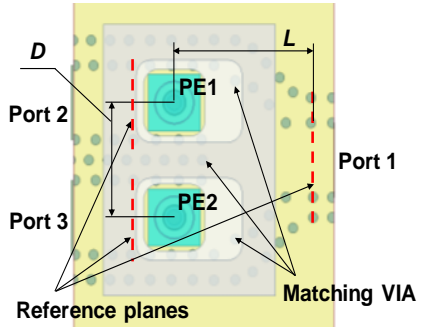

(a)



(b)
Fig. 4. (a) SPDT PE geometry, (b) E-field distribution at $79 \mathrm{GHz}$.

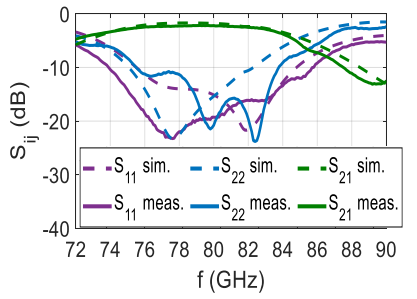

(a)



(b)
Fig. 5. $S$-parameters magnitudes of the SPDT PS: (a) State $1\left(\mathrm{PE}_{1}-\mathrm{OFF}\right.$-state, $\mathrm{PE}_{2}$ - ON-state); (b) State $2\left(\mathrm{PE}_{1}-\mathrm{ON}\right.$-state; $\mathrm{PE}_{2}-\mathrm{OFF}$-state).

$85.8 \mathrm{GHz}-10-\mathrm{dB}$ input impedance bandwidth with the peak insertion loss of $2.2 \mathrm{~dB}$; the isolation level is better than $30 \mathrm{~dB}$. The measured SPDT bandwidth is slightly narrower as compared to the SPST design due to an additional SIW discontinuity of the T-junction. For both PSs, there is an acceptable discrepancy with the simulations that is believed mainly due to PCB manufacturing tolerances $(\sim 25 \mu \mathrm{m})$ and assembling misalignments. A non-uniform PE volume conductivity distribution, realized in the experiments, can be another source of discrepancy.

In Table 1, we cross-compare the PS designs with the previously published SIW switches. As seen, most existing designs have been realized at lower frequencies that are intrinsically less challenging in terms of losses. Nonetheless, at mmWave frequencies, the proposed PSs demonstrate the lowest insertion loss, while preserving compactness and low profile.

\section{CONCLUSIONS}

The considered SIW PS concept realizes excellent electrical performance and excludes any mmWave-to-DC decoupling structures owing to the light actuation. The desired PS characteristics were achieved within the full W-band automotive radar bandwidth $(76-81 \mathrm{GHz})$. Further improvements of the proposed PS technology are possible through the integration of the PE with the LED or an optical fiber to simplify the design assembling and reduce optical power loss and total DC power consumption.

TABLE I

PERFORMANCE COMPARISON OF DIFFERENT SIW SWITCHES IMPLEMENTATIONS

\begin{tabular}{|l|c|c|c|c|c|}
\hline & {$[22]$} & {$[11]$} & {$[12]$} & {$[13]$} & This work \\
\hline Configuration & SPST/SPDT & SPDT & SPST & SPST & SPST/SPDT \\
\hline Implementation & Mechanical & PIN diodes & PIN diodes & Ferrite & PEs \\
\hline $\begin{array}{l}\text { Input }-10-d B \text { imp. } \\
\text { bandwidth, GHz }\end{array}$ & $75-110(38 \%)$ & $7-10.2(37 \%)$ & $20-25(22 \%)$ & $9.4-11.3(18 \%)$ & $72.5-89.5(21 \%) /$ \\
\hline Peak Ins. loss, dB & $7.5 / 10$ & 2.1 & 1.1 & 1.6 & $0.9 / 2.2$ \\
\hline Isolation, $\mathrm{dB}$ & $>50 / 50$ & $>10$ & $>10$ & $>27$ & $>25 />30$ \\
\hline $\begin{array}{l}\text { Area, } \lambda_{0}{ }^{2}\left(\lambda_{0}-\right. \\
\text { central wavelength) }\end{array}$ & $0.52 \times 0.54 / 0.62 \times 0.93$ & $0.92 \times 0.92$ & $0.4 \times 1.2$ & $0.44 \times 0.27$ & $0.37 \times 0.4 / 1 \times 0.97$ \\
\hline
\end{tabular}




\section{REFERENCES}

[1] H. H. Meinel, "Evolving automotive radar - From the very beginnings into the future", in Proc. 8th Eur. Conf. Antennas and Propagation (EuCAP 2014), 6-11 Apr. 2014, pp. 31073114.

[2] P. Kumari, J. Choi, N. Gonzalez-Prelcic, and R. W. Heath, "IEEE 802.11ad-based radar: An approach to joint vehicular communication-radar system," IEEE Transactions on Vehicular Technology, vol. 67, no. 4, pp. 3012-3027, Apr. 2018.

[3] K. Sakaguchi, T. Haustein, and S. Barbarossa, "Where, when, and how mmWave is used in $5 \mathrm{G}$ and beyond", IEICE Trans. Electron., vol. E100-C, no. 10, pp. 790-808, Oct. 2017.

[4] H. B. Sun, F. Brigui and M. Lesturgie, "Analysis and comparison of MIMO radar waveforms", in Proc. Int. Radar Conf., Oct. 2014, pp. 1-6.

[5] D. Zelenchuk, A. J. Martinez-Ros, and T. Zvolensky, "Wband planar wide-angle scanning antenna architecture", Journal of Infrared, Millimeter, and Terahertz Waves, vol. 34, issue 2, pp 127-139, Feb. 2013.

[6] F. Thome, E. Ture, P. Brückner, R. Quay, and O. Ambacher, "W-band SPDT switches in planar and tri-gate 100-nm gatelength GaN-HEMT technology", in Proc. German Microw. Conf., Mar. 2018, pp. 331-334.

[7] P. Song, R. L. Schmid, A. C. Ulusoy, and J. D. Cressler, "A high-power low-loss W-band SPDT switch using SiGe PIN diodes", 2014 IEEE Radio Frequency Integrated Circuits Symp. Dig., pp. 195-198, June 2014.

[8] J. Xu, Z. N. Chen, and X. Qing, "CPW center-fed singlelayer SIW slot antenna array for automotive radars", IEEE Trans. Antennas Propag., vol. 62, no. 9, pp. 4528-4536, Sep. 2014.

[9] J. Xu, W. Hong, H. Zhang, G. Wang, Y. Yu, and Z. H. Jiang, "An array antenna for both long- and medium-range $77 \mathrm{GHz}$ automotive radar applications", IEEE Trans. Antennas Propag., vol. 65, no. 12, pp. 7207-7216, Dec. 2017.

[10] A. Roev, R. Maaskant, A. Höök, and M. Ivashina, "Wideband mm-wave transition between a coupled microstrip line array and SIW for high-power generation MMICs", IEEE Microw.Wireless Compon. Lett., vol. 28, no. 10 , pp. $867-869$, Oct. 2018.

[11] I. Lim and S. Lim, "Substrate-integrated-waveguide (SIW) single-pole-double-throw (SPDT) switch for X-band applications," IEEE Microw. Wireless Compon. Lett., vol. 24, no. 8, pp. 536-538, Jun. 2014.

[12] A. B. Numan, J. F. Frigon, and J. J. Laurin, "Single-pole single-throw switch for substrate-integrated waveguide," IEEE Microw. Wireless Compon. Lett., vol. 28, no. 3, pp. 221-223, Feb. 2018.

[13] Q. D. Huang, Y. J. Cheng, and J. L. Li, "Surface-loaded ferrite substrate integrated waveguide switch," IEEE Microw. Wireless Compon. Lett., vol. 25, no. 4, pp. 232-234, Apr. 2015.

[14] C. D. Gamlath, D. M. Benton, and M. J. Cryan, "Microwave properties of an inhomogeneous optically illuminated plasma in a microstrip gap", IEEE Trans. Microw. Theory Techn., vol. 63, no. 2, pp. 374-383, Feb. 2015.

[15] E. Shepeleva, M. Makurin, A. Vilenskiy, and S. Chernyshev, "MM-wave patch antenna with embedded photoconductive elements for 1-bit phase shifting," in Proc. 2019 Photonics \& Electromagnetics Research Symposium - Spring, Rome, Italy, 2019, pp. 578-581

[16] A. W. Pang, C. D. Gamlath, and M. J. Cryan, "An optically controlled coplanar waveguide millimeter-wave switch," IEEE Microw. Wireless Compon. Lett., vol. 28, no. 8, 669671, Aug. 2018.

[17] M. A. Collet, C. D. Gamlath, and M. J. Cryan, "An optically tunable cavity-backed slot antenna," IEEE Trans. Microw. Theory Techn., vol. 65, no. 11, pp. 6134-6139, Nov. 2017.

[18] C. J. Panagamuwa, A. C. Chauraya, and J. C. Vardaxoglou, "Frequency and beam reconfigurable antenna using photoconducting switches," IEEE Trans. Microw. Theory Techn., vol. 54, no. 2, pp. 449-454, Feb. 2006.

[19] E. Shepeleva, M. Makurin, A. Lukyanov, A. R. Vilenskiy, S. L. Chernyshev, and M. V. Ivashina, "Low-loss K-band photoconductive switches in SIW technology", in Proc. 50th European Microwave Conference 2020 (EuMC 2020), 1214 Jan. 2021, pp. 538-541.

[20] T. F. Gallacher, "Optoelectronic modulation of mm-wave beams using a photo-injected semiconductor substrate," Ph.D. dissertation, the University of St. Andrews, UK, Mar. 2012.

[21] G. Dingemansm and W. M. M. Kessels, "Status and prospects of Al2O3-based surface passivation schemes for silicon solar cells," Journal of Vacuum Science \& Technology, A 30 (4), 040802, 2012.

[22] S. Wei, C. H. Yang, Y. C. Chen, T. A. Chen, and C. Y. Chang, "V- and W-band substrate integrated waveguide (SIW) mechanical switch," IEEE Trans. Microw. Theory Techn., vol. 66, no. 6, pp. 3090-3098, Jun. 2018. 